\title{
Factores relacionados con la cirugía fallida de hernia discal lumbar
}

\author{
J. Rodríguez-García; A. Sánchez-Gastaldo; T. Ibáñez-Campos; C. Vázquez-Sousa; M. Cantador-Hornero; J.A. \\ Expósito-Tirado; A. Cayuela-Domínguez y C. Echevarría-Ruiz de Vargas
}

Servicio de Rehabilitación. Hospitales Universitarios Virgen del Rocío. Sevilla.

\section{Resumen}

Introducción. La cirugía descompresiva de hernia discal es la intervención quirúrgica más frecuente a nivel de la columna lumbar. La cirugía fallida oscila entre un 10 y un $40 \%$ de los casos, constituyendo el denominado Síndrome de la Cirugía Raquídea Fracasada (SCRF). La reincorporación laboral a medio plazo tras la cirugía se sitúa en torno al $\mathbf{7 0 - 8 5 \%}$, y existen pocos estudios que analicen la calidad de vida tras la intervención. Los objetivos de este estudio son conocer la incidencia de cirugía fallida de hernia discal lumbar en nuestro medio, identificando aquellos factores que puedan influir en su aparición; y estudiar factores ambientales como la reincorporación laboral y la calidad de vida a medio plazo tras esta cirugía, así como su relación con el éxito o fracaso quirúrgico.

Material y métodos. Estudio descriptivo transversal de 117 pacientes intervenidos de hernia discal lumbar durante el primer semestre del 2003 en nuestro hospital. Para valorar la incidencia de cirugía fallida y factores relacionados se han recogido datos clínicos de la Historia Clínica de los pacientes de la muestra. Y, asimismo, se ha entrevistado telefónicamente a 91 de ellos -los que contestaron- para valorar factores no clínicos relacionados con el SCRF, tales como reincorporación laboral, satisfacción con la cirugía, realización de tratamiento rehabilitador y calidad de vida a través del Cuestionario de Salud SF-36. Para el análisis de resultados se ha empleado el programa estadístico SPSS 11.01.

Resultados. En una muestra en la que la proporción ente ambos sexos es de 1/1 y la edad media de 45 [35-54] años, con una clínica más frecuente de ciática derecha, de más de 6 meses de evolución, correlacionada con el hallazgo radiológico de hernia L5-S1, un 37,9\% ha presentado el SCRF. Aunque es escaso el número de pacientes que en nuestra serie afrontan una reintervención, se ha observado en ellos una incidencia del SCRF notablemente superior $(52,2 \%)$ a los que se someten a una

Recibido: 18-08-04. Aceptado: 01-02-05. primera cirugía (32\%). Los factores clínicos predictivos de resultados desfavorables en pacientes sometidos a primera cirugía han sido la clínica precirugía de ciática bilateral, el hallazgo de estenosis de canal asociada a hernia discal y la comorbilidad; mientras que los sociolaborales han sido pacientes sin estudios y aquellos que trabajan en los sectores de la conducción, construcción y hostelería. La reincorporación laboral se produjo en el $64 \%$ de los trabajadores activos previamente. Sólo en torno al $10 \%$ se encuentran insatisfechos con la opción quirúrgica, relacionándose significativamente tanto esto como las dimensiones de función física, dolor, vitalidad y rol emocional en el SF-36 con el SCRF.

Conclusiones. Uno de cada 3 pacientes intervenidos de hernia discal lumbar en nuestro medio presenta cirugía fallida, reincorporándose laboralmente 2 de cada 3 pacientes previamente activos.

El paciente con cirugía fallida se encuentra afectado por dolor, hasta el punto de repercutir y limitar las actividades del hogar y las laborales. Además presenta frecuentemente sensación de cansancio o agotamiento, y problemas emocionales que interfieren tanto en las actividades de la vida diaria como en el trabajo.

PALABRAS CLAVE: Hernia discal lumbar. Cirugía fallida. Reincorporación laboral. Calidad de vida.

Related factors with the failed surgery of herniated lumbar disc

Summary

Introduction. The surgery for herniated disc is the most common operation at the level of the lumbar spine. The failed surgery rates range between $10 \%$ and $40 \%$, conforming what is known as Failed Back Surgery Syndrome (FBSS).

Return to work after surgery occurs in $70-85 \%$ of

Abreviaturas: FBSS: Failed Back Surgery Syndrome. SCRF: sindrome de la cirugía de raquis fallida. 
the cases. There are a few studies analysing the quality of life after the operation. The aims of this study are to know the incidence of the herniated disc lumbar failed surgery in our area, identify those factors influencing its development, and study behavioural parameters as the return to work and the quality of life at a middle term after surgery, also its relation with the success or failure surgery.

Material and methods. A descriptive transversal study of 117 patients operated for herniated lumbar disc during the first six months of the year 2003 is reported. In order to evaluate the incidence of failed surgery and the related factors, the clinical records were retrospectively analyzed. Ninety one patients were interviewed by phone using the Health Questionnaire SF-36, in order to analyze the non clinical factors related to FBSS, such as labour reincorporation, satisfaction with surgery, realization of rehabilitation treatment and quality of life after surgery. For the statistical analysis of the results, we used the program SPSS 11.01.

Results. In a sample in which the proportion between both sexes was $1 / 1$, and the middle age was over 45 years [35-54], in which the most frequent clinical symptom was right sciatica, lasting more than 6 months, correlated to disc herniation at L5-S1 level, 37'9\% of the patients presented FBSS. Although there were a few patients with reoperation in our study, the incidence of FBSS in these patients was higher $(52,9 \%)$ than in patients who suffered this surgery for first time $(32 \%)$. The predictive clinical factors of an unfavourable result in patients operated on for first time were bilateral sciatica, the presence of stenosis associated to herniated disc and comorbidity factors. On the other hand the sociolabor factors identified were a low culture level and those working as drivers, building and service sectors. Return to work occurred in the $64 \%$ of the active workers before surgery. Only around $10 \%$ of patients were dissatisfied with surgical result and there was a significant relationship between this and the physical function, pain, vitality and emotional status in the SF-36 with the FBSS.

Conclusions. One out of three patients operated of herniated lumbar disc in our area presented failed disc surgery and the return to work occurred in 2 out of three patients active before the operation. The failed surgery patient suffers from pain, that interferes and limits the labor and home activities. Furthermore, the patient presents frequent sensation of fatigue and exhaustion and also emotional problems that contribute to interfere with work and activities of the daily life.

KEY WORDS: Herniated disc surgery. Failed surgery. Return to work. Quality of life.

\section{Introducción}

La cirugía de descompresión de la hernia discal lumbar es la intervención quirúrgica más frecuente desarrollada a nivel de la columna vertebral, y una de las más comunes en cirugía ortopédica. Está representada en su grado máximo en países como Estados Unidos con un número aproximado de 200.000 al año ${ }^{3,20}$, donde es al menos un $40 \%$ superior al resto de los países, según mostró un estudio comparativo internacional del porcentaje de cirugía de hernia discal lumbar ${ }^{17}$. No se disponen de datos oficiales en España, aunque se cifra en torno a las 3.500 intervenciones al año.

El éxito de esta cirugía varía en un amplio rango, encontrándose en la literatura porcentajes desde el 60 al $90 \%$, constituyendo el porcentaje restante el denominado Síndrome de la Cirugía Raquídea Fracasada (SCRF) o Failed Back Surgery Syndrom ${ }^{17}$. Repetidos estudios sugieren que la recurrencia del dolor tras la cirugía, en ausencia de patología del disco u ósea que lo justifique, tiene como principal etiología (en torno al $24 \%$ de los casos) la fibrosis peridural $^{19}$, aunque su asociación con la recurrencia del dolor es un tema muy debatido, ya que la fibrosis peridural postquirúrgica es un efecto adverso inevitable ${ }^{14,19,22}$. Existe una fuerte evidencia de que factores psicosociales pueden tener una significativa influencia en la percepción y cronicidad del dolor, lo que podría explicar parcialmente porqué esta cirugía no es exitosa, incluso cuando los problemas morfológicos han desaparecido tras la intervención ${ }^{20}$. Debido al alto porcentaje de cirugía fracasada, las reintervenciones quirúrgicas tras la primera cirugía se hacen necesaria, en un rango que oscila entre el 5-18\% de los pacientes, obteniendo en estos casos pobres resultados respecto a la disminución del dolor y mejoría funcional ${ }^{11}$.

La reincorporación laboral de estos pacientes, transcurrido 1 año de la cirugía, oscila entre el 70 y el 85\%, aunque en la literatura médica encontramos estudios a largo plazo con una reincorporación al mismo puesto de trabajo que varía entre el 65 y 98\% ${ }^{5,21}$. Es fácil suponer la repercusión socioeconómica que supone esta cirugía en un Estado, ya que a las bajas laborales pre y postcirugía, debemos añadir un porcentaje nada despreciable de pensiones por invalidez, concedidas a estos pacientes tras el fracaso quirúrgico.

Por último, al margen de la reincorporación laboral, un aspecto poco estudiado en los distintos artículos revisados sobre los resultados de la cirugía de hernia discal lumbar es la calidad de vida del paciente tras la cirugía, y si ésta mejora significativamente respecto al grupo de pacientes no intervenidos con la misma patología.

Los objetivos propuestos con este trabajo son:

a) Conocer la incidencia de cirugía fallida de hernia discal lumbar en nuestro medio, identificando aquellos factores que puedan influir en su aparición, 
Tabla 1

Entrevista lelefónica realizada a los pacientes de la muestra

\begin{tabular}{|c|c|c|}
\hline 1.- Estado Civil & $\begin{array}{l}\text { Soltero } \\
\text { Casado } \\
\text { Separado } \\
\text { Divorciado } \\
\text { Viudo }\end{array}$ & \\
\hline 2.- Nivel de estudios & $\begin{array}{l}\text { Sin estudios } \\
\text { Primarios } \\
\text { Secundarios } \\
\text { Formación profesional } \\
\text { Estudios medios } \\
\text { Universitarios } \\
\text { Otros no reglados }\end{array}$ & \\
\hline 3.- Situación laboral precirugía & $\begin{array}{l}\text { Activo laboralmente } \\
\text { Jubilado/pensionista (antes trabajando) } \\
\text { Pensionista (antes ama de casa) } \\
\text { Parado } \\
\text { Estudiante } \\
\text { Otros }\end{array}$ & $\begin{array}{l}\text { Si activo laboralmente: } \\
\text { - Tipo de trabajo } \\
\text { - Carga peso habitualmente } \\
\quad * \text { Si } \\
\quad * \text { No }\end{array}$ \\
\hline 4.- Situación laboral postcirugia & $\begin{array}{l}\text { Mismo trabajo/situación } \\
\text { Trabaja a un nivel inferior } \\
\text { Parado } \\
\text { Invalidez }\end{array}$ & $\begin{array}{l}\text { Si reincorporación laboral, } \\
\text { meses de baja tras cirugía: } \\
\qquad \begin{aligned}->3 \text { meses } \\
-3 \text { sem }-3 \text { meses } \\
->3 \text { meses }\end{aligned}\end{array}$ \\
\hline 5.- Rehabilitación postcirugía & $\begin{array}{l}\text { No } \\
\mathrm{Si}\end{array}$ & $\begin{array}{l}\text { En caso afirmativo, } \\
\text { ¿Cuanto tiempo? }\end{array}$ \\
\hline 6.- Satisfacción & $\begin{array}{l}\text { ¿Si pudiera regresar al momento antes de } \\
\text { la intervención,volvería a elegir } \\
\text { la opción quirúrgica? }\end{array}$ & $\begin{array}{l}-\mathrm{Si} \\
\text { - No } \\
\text { - No sabe/ No contesta }\end{array}$ \\
\hline 7.- Calidad de vida & Cuestionario de Salud SF-36 & \\
\hline
\end{tabular}

${ }^{1}$ Transportar 10 ó más kilos con los miembros superiores diariamente

diferenciando entre los que se intervienen por primera vez y los que se someten a reintervención.

b) Estudiar factores ambientales tales como la reincorporación laboral y la calidad de vida a medio plazo tras esta cirugía, así como su relación con el éxito o fracaso quirúrgico.

\section{Material y métodos}

Estudio descriptivo transversal de los 138 pacientes intervenidos quirúrgicamente de hernia discal lumbar durante el primer semestre del año 2003 en nuestro hospital. Tras solicitar todas estas historias clínicas a la Unidad de Documentación Científica sin criterios de selección, se ha conseguido acceder a 117 de éstas, que han conformado la muestra del estudio, no encontrándose en los archivos las 21 historias restantes en el momento de su solicitud.

La recogida de datos, tanto sociodemográficos como de la propia patología, se ha llevado a cabo mediante un cuestionario con los siguientes ítems: edad en el momento de la intervención, sexo, antecedentes personales de interés, primera cirugía o reintervención quirúrgica (en caso 
de reintervención, el tiempo transcurrido en meses desde la última cirugía), tiempo de evolución del dolor previo a la cirugía (menos de 3 meses, entre 3 y 6 meses, y más de 6 meses), nivel de la hernia discal (L2-L3, L3-L4, L4-L5, L5-S1 o doble hernia L4-L5/L5-S1), sintomatología previa a la cirugía (lumbociatalgia izquierda, derecha o bilateral, o lumbalgia sin irradiación), complicaciones postquirúrgicas, persistencia del mismo dolor tras la intervención quirúrgica, reaparición del mismo dolor tras la intervención (en caso afirmativo se ha distinguido entre reaparición con menos de 1 mes, entre 1 y 3 meses, y más de 3 meses), si se solicitó Resonancia Magnética (RM) tras la persistencia del dolor o la reaparición del dolor, y el resultado de la misma en base al informe del radiólogo (fibrosis peridural, reaparición de hernia discal al mismo nivel, fibrosis peridural más reaparición de la misma hernia, hernia discal a otro nivel o si no se encontraron hallazgos que justificasen la reaparición del dolor).

En una segunda parte, se ha entrevistado telefónicamente a estos pacientes para valorar fundamentalmente la reincorporación laboral y la calidad de vida a medio plazo (entre 6 y 12 meses tras la cirugía); aunque también recogimos el grado de satisfacción con la cirugía, si siguieron algún programa terapéutico médico-rehabilitador, estado civil y nivel de estudios. Se ha conseguido contactar telefónicamente con 91 de los 117 (78\%). Las preguntas que se formularon en esta entrevista están recogidas en la tabla 1. Para valorar calidad de vida se ha seleccionado el cuestionario Medical Outcomes Study Short-Forme 36 (SF-36) en su versión española previamente validada, conocida como Cuestionario de Salud SF-36 ${ }^{1}$. Contiene 36 ítems divididos en las siguientes áreas:

1.- Estado funcional: representado por las siguientes dimensiones; función física, función social, limitaciones del trabajo por problemas físicos y limitaciones del mismo por problemas emocionales.

2.- Bienestar emocional: incluye las dimensiones salud mental, vitalidad y dolor.

3.- Percepción de la salud general: valoración personal de la salud actual, perspectivas de salud en el futuro y resistencia a enfermar.

Los ítems y las dimensiones del SF-36 están puntuados de forma que, a mayor puntuación mejor es el estado de salud. Para cada dimensión, los ítems son codificados, agregados y transformados en una escala que tiene un recorrido desde 0 (el peor estado de salud para esa dimensión) hasta 100 (el mejor estado de salud). Es uno de los instrumentos genéricos más utilizados en la medición de la calidad de vida relacionada con la salud, pudiendo realizarse de forma autoadministrada o mediante entrevista personal o telefónica ${ }^{4}$.

El análisis estadístico se ha realizado utilizando el programa estadístico SPSS 11.01. Las variables cualitativas se han expresado mediante frecuencias absolutas y relativas, mientras que para las cuantitativas, al no seguir una distribución normal, se ha utilizado percentiles. La comparación de las variables cualitativas se ha realizado con la prueba Chi-cuadrado. Para la comparación de variables cuantitativas según una variable cualitativa dicotómica se ha utilizado la U de MannWhitney, y el test de Kruskal-Wallis cuando la variable cualitativa tenía más de 2 categorías.

\section{Resultados}

Tras haber comprobado que los 26 pacientes que no respondieron telefónicamente, no presentaban diferencias estadísticamente significativas con los 91 que pudimos contactar, en relación al sexo, edad, lugar de residencia, clínica precirugía, tiempo de evolución del dolor antes de la intervención etc..., se ha realizado un análisis descriptivo de los 117 pacientes de la muestra, excepto en aquellos datos que obtuvimos telefónicamente, como el nivel de estudios, estado civil, situación laboral, satisfacción, rehabilitación y calidad de vida, que se ha llevado a cabo sólo con los 91 contactados. En un segundo paso, se ha relacionado todas las variables con la cirugía fallida, entendida como la persistencia y/o reaparición del mismo dolor tras la cirugía.

La muestra ha quedado formada por 59 varones $(50,4 \%)$ y 58 mujeres $(49,6 \%)$, con una edad media, expresada en percentiles, de P50 [P25-P75], de 45 [35-54] años. En la tabla 2 se han mostrado los datos relacionados con la hernia de disco lumbar, exponiendo en negrita los porcentajes más altos o que consideramos de mayor interés. Se ha de resaltar que existen algunos casos donde no se recogían con claridad determinados aspectos de la patología en las historias clínicas de los pacientes, y que por tanto, no se han incluido, por ejemplo, dos pérdidas en relación a la clínica precirugía, 1 pérdida en el tiempo de evolución previo a la intervención, etc...; por lo que no debe sorprender que al contabilizar el total de pacientes en algunas variables de la tabla no se encuentren los 117 pacientes.

En relación a la persistencia y reaparición del dolor, hemos de destacar que existen 5 pacientes que se han incluido en ambos grupos ya que presentaron persistencia

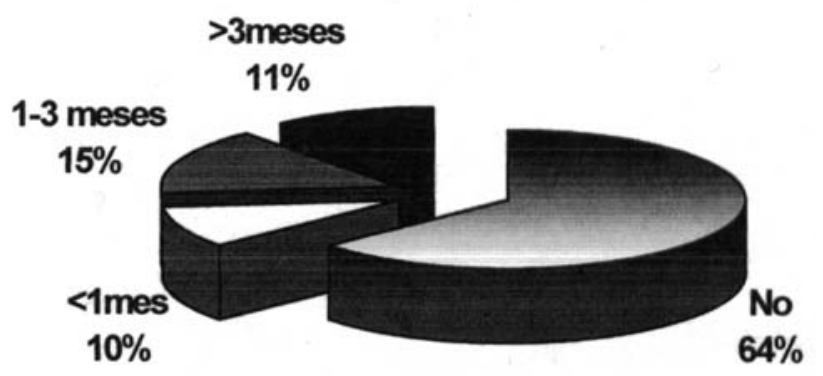

Figura 1. Reaparición del dolor tras la cirugía, según el período de tiempo de aparición del mismo. 
Tabla 2

Datos relacionados con la cirugía de hernia discal lumbar tanto precirugía y tras la Intervención

\begin{tabular}{|c|c|c|c|c|c|}
\hline Variables & $\mathrm{N}^{\circ}$ pacientes & $\%$ & Variables & cientes & $\%$ \\
\hline Clínica precirugía & & & Primera cirugía & & \\
\hline Ciática izquierda & 44 & 37,6 & $\mathrm{Si}$ & 94 & 80,3 \\
\hline Ciática derecha & 59 & 50,4 & No (reintervención) & 23 & 19,7 \\
\hline Ciática bilateral & 11 & 9,4 & & & \\
\hline \multirow[t]{2}{*}{ Lumbalgía } & 1 & 0,85 & Complicaciones & & \\
\hline & & & No & 107 & 91,4 \\
\hline Tiempo evolución del dolor & & & Tempranas & 7 & 6 \\
\hline$<3$ meses & 27 & 23,1 & Tardías & 3 & 2,6 \\
\hline Entre 3 y 6 meses & 29 & 24,8 & & & \\
\hline \multirow[t]{2}{*}{$>6$ meses } & 60 & 52,7 & Persistencia del dolor & & \\
\hline & & & No & 105 & 89,75 \\
\hline Nivel de la hernia & & & $\mathrm{Si}$ & 12 & 10,25 \\
\hline L2-L3 & 1 & 0,9 & & & \\
\hline L3-L4 & 5 & 4,3 & Reaparición del dolor & & \\
\hline L4-L5 & 46 & 39,6 & No & 79 & 68,1 \\
\hline L5-S1 & 61 & 52,1 & $\mathrm{Si}$ & 37 & 31,9 \\
\hline \multirow[t]{2}{*}{ L4-L5/L5-S1 } & 4 & 3,4 & & & \\
\hline & & & Resonancia magnética & & \\
\hline \multicolumn{2}{|l|}{ Otras alteraciones raquídeas } & & No & 90 & 76,9 \\
\hline No & 101 & 86,3 & $\mathrm{Si}$ & 27 & 23,1 \\
\hline Estenosis de canal & 14 & 12 & Fibrosis peridural & 11 & $40,7 *$ \\
\hline Listesis & 1 & 0,85 & Recidiva hemiaria & 8 & $29,6 *$ \\
\hline Estenosis + listesis & 1 & 0,85 & Fibrosis + recidiva & 5 & $18,5^{*}$ \\
\hline
\end{tabular}

*Estos porcentajes son respecto del total de ResonancíaMagnétícas realizadas, y no sobre el total de la muestra

del dolor tras la cirugía, que desapareció, para posteriormente reaparecer. En la figura 1 se ha representado la reaparición del dolor tras la cirugía, subdividido en los intervalos de tiempo en que se produjo ésta.

El estado civil más representado en los pacientes ha sido el casado (72,5\%), seguido de los solteros $(13,2 \%)$ y los separados (7,7\%), encontrando sólo 3 casos de divorciados y viudos. Respecto al nivel de estudios, un 44,9\% presentaban estudios primarios seguido de aquellos sin estudios que conformaban el $24,2 \%$ de la muestra; con porcentajes sensiblemente inferiores se ha encontrado los pacientes con formación profesional y estudios universitarios $(7,7 \%)$, y aquellos con estudios medios $(2,2 \%)$.

En lo relativo a la reincorporación laboral, se ha encontrado que antes de la intervención 62 pacientes se encontraban activos laboralmente $(68,1 \%), 14$ eran pensionistas $(15,4 \%), 11$ amas de casa $(12,1 \%), 1$ parado $(1,1 \%), 1$ estudiante $(1,1 \%)$ y en 2 casos otra situación $(2,2 \%)$. 


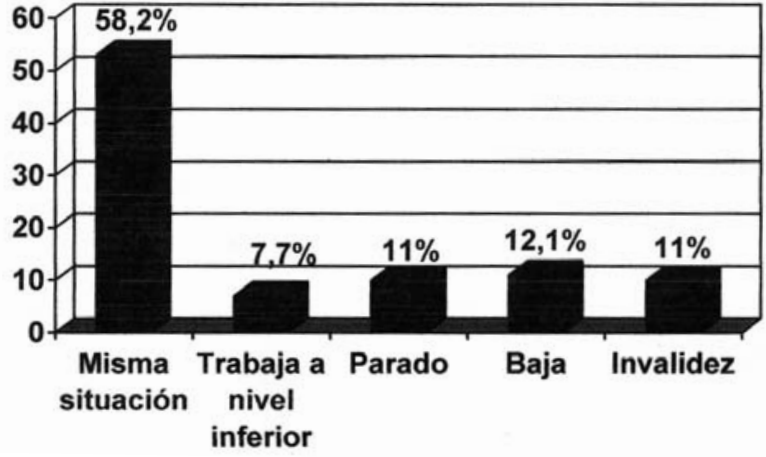

Figura 2. Situación laboral tras la cirugía expresado en porcentajes.

Analizando los 62 pacientes que se encontraban trabajando fuera de casa, en 15 casos se trataba de trabajos agrícolas $(24,2 \%), 6$ eran conductores $(9,7 \%), 6$ trabajadores en el área de la hostelería $(9,7 \%), 4$ pacientes trabajaban en la construcción $(6,5 \%)$ y los 31 restantes ejercían profesiones encuadradas en el grupo de otras. De los 62 pacientes que trabajaban, $32(58,1 \%)$ cargaban peso de forma habitual en el trabajo. La situación laboral tras la cirugía se ha presentado en la figura 2 .

De los 62 pacientes que trabajaban fuera de casa, 40 llegaron a reincorporarse laboralmente $(64,5 \%)$, y de estos 40 pacientes, 7 lo hicieron en menos de 3 semanas (17,5\%), 11 entre 3 semanas y 3 meses $(27,5 \%)$ y 22 se reincorporaron después de 3 meses (55\%).

En relación a las 10 complicaciones postquirúrgicas $(8,6 \%), 7$ fueron tempranas (un proceso febril, 2 infecciones del tracto urinario, una retención urinaria, un síndrome de cola de caballo y 2 infecciones de la herida quirúrgica) y 3 tardías (una trombosis venosa profunda y 2 fístulas de líquido cefalorraquídeo).

En el apartado de tratamiento médico-rehabilitador tras la cirugía, 30 pacientes (33\%) llevaron a cabo algún tipo de tratamiento, que en la mayoría de los casos consistió en un programa de capacitación muscular y/o electroterapia, siendo en un $56,7 \%$ de los casos por un período aproximado de un mes, y un $30 \%$ por un tiempo de 2 meses. Respecto a la satisfacción con el proceso quirúrgico, 72 pacientes $(79,1 \%)$ afirmaron que elegirían de nuevo la opción quirúrgica si volvieran al momento previo a la intervención, 10 no la elegirían de nuevo (11\%) y 9 pacientes no sabrían que elegir (9,9\%). La comorbilidad de los pacientes de la muestra se ha representado en la figura 3.

Tras este análisis descriptivo, se han relacionado las distintas variables de la muestra con cirugía fallida de hernia discal lumbar, para lo cual se han agrupado todos aquellos pacientes con persistencia y/o reaparición del dolor en un solo grupo, encontrando que 44 pacientes $(37,9 \%)$ de la muestra presentaron el SCRF. Sin embargo, por las peculiaridades de los pacientes intervenidos por primera vez de hernia discal lumbar y aquellos reintervenidos, se han analizado de forma independiente para establecer relaciones con el SCRF.

Primera cirugía: La incidencia del SCRF de los 94 pacientes intervenidos por primera vez fue de un $32 \%$, siendo los resultados de la relación de las distintas variables con cirugía fallida los siguientes:

- Sexo: Se han encontrado porcentajes discretamente superiores de cirugía fallida en varones $(35,6 \%$ frente al $32,7 \%$ ).

- Edad: No se observan diferencias respecto a la edad expresada en percentiles entre los pacientes con cirugía fallida $46[36-53,75]$ y aquellos que no la presentaron 46[34-55,50].

- Clínica precirugía: El SCRF ha aparecido más frecuentemente en la ciática bilateral $(50 \%)$, seguida de la ciática derecha $(38,8 \%)$, presentando la ciática izquierda $(24,2 \%)$ el menor porcentaje del SCRF.

- Tiempo de evolución del dolor precirugía (en

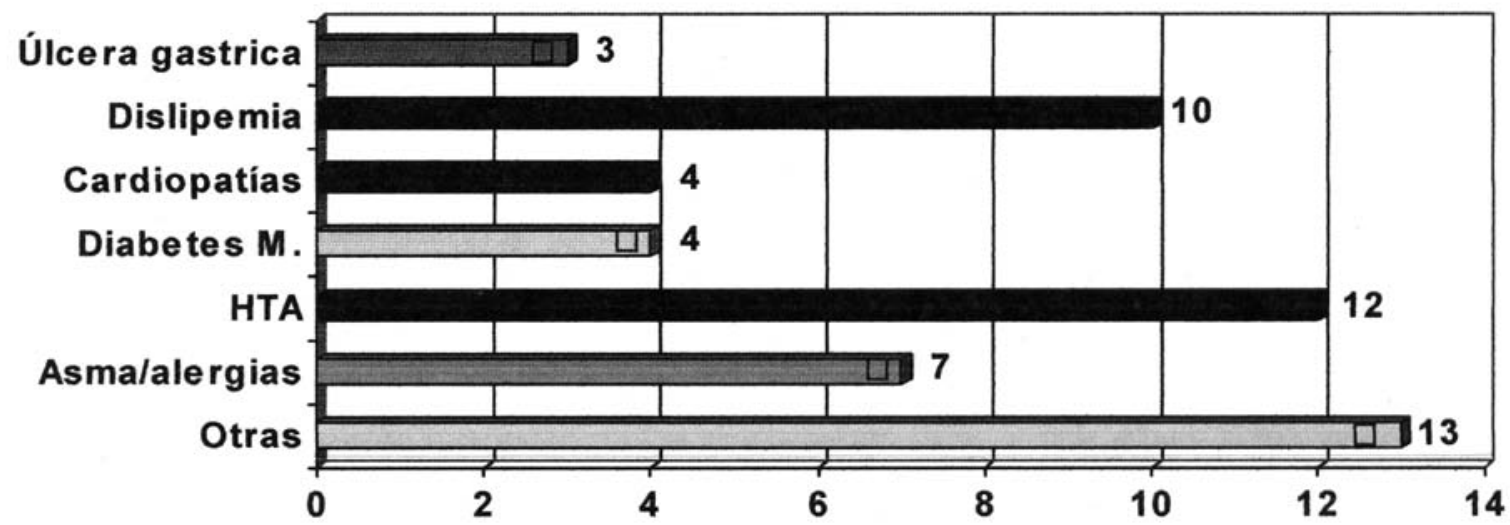

Figura 3. Patologías asociadas de los pacientes de la muestra expresadas en números de casos. Nota al pie de figura: Diabetes M: Diabetes Mellitus; HTA: Hipertensión arterial. 
meses): Se han encontrado diferencias entre los pacientes con y sin SCRF respecto al tiempo de evolución del dolor; los pacientes con un tiempo de evolución del dolor superior a 6 meses han presentado un $37,7 \%$ de cirugía fallida frente al 33,3\% y $27,3 \%$ de aquellos con un tiempo de evolución de 3 a 6 meses y menor de 3 meses respectivamente.

- Nivel de la hernia: A nivel L4-L5 el SCRF ha sido de un $40 \%$ frente al $33,3 \%$ de la producida a nivel L5-S1. Resulta llamativo que ninguno de los 3 pacientes con doble hernia L4-L5/L5-S1 presentara el SCRF, aunque es muy escaso el número de pacientes así como en los otros niveles.

- Otras alteraciones raquídeas: Aunque es escaso el número de pacientes que las presentaban (16 casos), se ha encontrado que un $58,3 \%$ de los pacientes con estenosis de canal lumbar presentó el SCRF, frente al 30\% de los que no presentaban otras alteraciones raquídeas.

- Complicaciones postquirúrgicas: Un solo caso de los 9 pacientes con complicaciones presentó el SCRF.

- Hallazgos radiológicos: No se ha objetivado ninguna relación en los pacientes de la muestra que presentaron recidiva herniaria en RM con la cirugía fallida, y una débil relación de ésta con la aparición de fibrosis peridural en aquellos en que se realizó una RM.

- Estado civil: Porcentaje ligeramente superior del SCRF en los casados $(43,1 \%)$ frente a los separados $(40 \%)$ y solteros $(36 \%)$. No se ha encontró ningún caso en divorciados y viudos.

- Nivel de estudios: Se ha encontrado un $50 \%$ del SCRF en pacientes sin estudios y un $42,9 \%$ en aquellos con estudios primarios y universitarios, frente a aquellos con estudios secundarios y formación profesional que presentaron porcentajes de 18,8 y $20 \%$ respectivamente.

- Situación laboral previa: El 40,8\% en los activos laboralmente y el $37,5 \%$ de los pensionistas han presentado el SCRF frente al $11,1 \%$ de las amas de casa. Respecto al tipo de trabajo, en los activos laboralmente destaca el $75 \%$ del SCRF en los conductores, frente al 50\% de los trabajadores de los sectores de la construcción y hostelería, y el $42,9 \%$ del sector agrícola. No se ha encontrado relación con cargar peso en el trabajo, siendo menor incluso el porcentaje del SCRF en los que afirmaban cargar peso $(41,4 \%)$ frente a los que no (40\%).

- Situación laboral postcirugía: Destaca el 83,3\% y el $66,7 \%$ del SCRF en los que se encontraban de baja y en situación de invalidez respectivamente tras 6-12 meses de la cirugía, frente a los parados $(44,4 \%)$, a los que se encontraban activos laboralmente en la misma situación $(30,4 \%)$ y a los que trabajaban en un nivel inferior (16,7\%).

- Meses de baja tras la cirugía: En aquellos que se reincorporaron laboralmente se observa que el periodo de baja laboral que ha presentado un mayor porcentaje de cirugía fallida ha sido el superior a 3 meses $(36,8 \%)$, frente al periodo menor de 3 semanas $(28,6 \%)$ y el comprendido entre 3 semanas y 3 meses $(20 \%)$.

- Rehabilitación: Un 57,9\% de los que hicieron un programa rehabilitador presentaron el SCRF, frente al 32,1\% de los que no la realizaron.

- Satisfacción: Un 100\% de los pacientes insatisfechos con la intervención presentó el SCRF frente al 30,5\% de los satisfechos, siendo este resultado estadísticamente significativo. En los pacientes que no sabían si volverían a intervenirse apareció en un 50\% el SCRF.

- Comorbilidad: Se ha encontrado mayor porcentaje del SCRF en pacientes con diabetes y cardiopatía (75\% frente al 36,8\% que no la padecían, en ambos casos), con hipertensión (66,7\% frente al $34,9 \%$ que no presentaban hipertensión), con dislipemia ( $75 \%$ frente al $35,4 \%$ que no, significativo estadísticamente) y en el grupo de otras enfermedades (53,8\% frente al $41 \%$ de los que no).

Reintervención: La incidencia del SCRF de los 23 pacientes reintervenidos de hernia discal lumbar fue de un 52,2\%. Una vez relacionadas las distintas variables con cirugía fallida, describimos únicamente los resultados más significativos encontrados dado el escaso volumen de pacientes.

Sexo: Se han observado porcentajes claramente superiores de cirugía fallida en los 14 varones $(64,3 \%)$ frente a las 9 mujeres $(33,3 \%)$.

- Edad: Notablemente superior en los pacientes con el SCRF (44[38-57] frente a los que no la presentaron (39 [3349]).

- Tiempo de evolución del dolor precirugía (en meses): Se han encontrado notables diferencias entre los pacientes con y sin SCRF respecto al tiempo de evolución del dolor; los pacientes con un tiempo de evolución del dolor entre 3 y 6 meses han presentado un $85,7 \%$ de cirugía fallida frente al $44,4 \%$ y $28,6 \%$ de aquellos con un tiempo de evolución menor de 3 meses y mayor de 6 meses respectivamente.

- Nivel de estudios: se ha encontrado un $90,9 \%$ del SCRF de los 11 pacientes con estudios primarios, el resto de niveles han presentado un mínimo número de pacientes como para ser analizados.

- Situación laboral postcirugía: Destaca el 75\% y $80 \%$ de cirugía fallida en aquellos que se encontraban en situación de invalidez y de baja respectivamente, frente al $42,9 \%$ de los que se encontraban activos laboralmente en la misma situación.

- Satisfacción: Un 100\% de los pacientes insatisfechos con la intervención presentó el SCRF frente al 46,2\% de los satisfechos. En los pacientes que no sabían si volverían a intervenirse apareció en un $66,7 \%$ el SCRF.

Para conocer la afectación de estos pacientes en su calidad de vida se ha relacionado en sus distintas dimensiones con aquellos que presentaron el SCRF, sin diferenciar entre $1^{a}$ cirugía o reintervención. Se han mostrado los resultados 
Tabla 3

Resultados de la relación entre las distintas dimensiones del SF-36 con la cirugía fallida

\begin{tabular}{|l|c|c|}
\hline DIMENSIONES SF-36 & $\begin{array}{l}\text { U de Mann- } \\
\text { Whitney }\end{array}$ & $\begin{array}{l}\text { Significación } \\
\text { Estadística }\end{array}$ \\
\hline Función física & 725,0 & $\mathbf{, 0 2 0}$ \\
\hline Rol físico & 824,0 &, 106 \\
\hline Dolor corporal & 586,0 & $\mathbf{, 0 0 1}$ \\
\hline Salud general & 836,5 &, 154 \\
\hline Vitalidad & 761,0 & $\mathbf{, 0 4 2}$ \\
\hline Función social & 813,5 &, 078 \\
\hline Rol emocional & 758,5 & $\mathbf{, 0 1 8}$ \\
\hline Salud mental & 775,5 &, 055 \\
\hline
\end{tabular}

en la tabla 3, señalándose en negrita los resultados significativos estadísticamente.

\section{Discusión}

En los pacientes de la muestra intervenidos de hernia discal lumbar se ha encontrado una igualdad entre ambos sexos, con una proporción $1 / 1$, a diferencia de otros estudios donde aparece discreta prevalencia por el sexo masculino, entre un 60 y un 75\% ${ }^{2-6,11,19}$. En relación a la edad, la media de la muestra expresada en percentiles es de 45 años [35-54], oscilando las edades medias de los artículos revisados entre 37 y 49 años ${ }^{2,5,6,19}$.

Respecto a los datos de la patología discal, los pacientes de nuestro estudio han presentado con mayor frecuencia ciática derecha $(50,42 \%)$ respecto a la ciática izquierda $(37,6 \%)$ y a la ciática bilateral $(9,4 \%)$, encontrando sólo un estudio donde se específica la irradiación unilateral (88\%) y bilateral $(10 \%)^{6}$; en el resto de los artículos revisados no se detallan estos datos. En relación al tiempo de evolución del dolor previo a la cirugía, se han seleccionado los intervalos de tiempo de menos de 3 meses, entre 3 y 6 meses, y más de 6 meses, considerando los distintos artículos revisados, para tratar de establecer una relación entre este parámetro y el éxito de la intervención ${ }^{16,20}$; en este sentido se ha encontrado que el $52,7 \%$ de los pacientes de la muestra referían haber presentado sintomatología dolorosa durante más de 6 meses antes de la intervención, coincidiendo con el estudio de Schade et al. ${ }^{20}$, que en su estudio prospectivo con 46 pacientes, halló un $54 \%$ de los mismos. Por último, el nivel de hernia más afectado en el trabajo fue el L5-S1 con un $53,8 \%$, seguido por el nivel L4-L5 con un 39,6\%, siendo estos los niveles más frecuentemente afectados en las distintas series revisadas con porcentaje similares ${ }^{2,6,11}$.

Aunque la mayor parte de los estudios revisados se centran en la primera cirugía, excluyendo las reintervenciones, se ha considerado interesante valorar la diferencia de resultados entre pacientes que se intervienen por primera vez y aquéllos que han sido expuestos a cirugía más de una vez, por afectación discal al mismo nivel, encontrando que un $20,9 \%$ de nuestros pacientes se volvían a someter a cirugía de hernia discal, algo superior a los estudios encon$\operatorname{trados}^{7,11}$, donde oscila entre el 5 y el $15 \%$. Las complicaciones (tempranas y tardías) que se han encontrado fueron, exceptuando 2 casos, leves-moderadas y se dieron en un porcentaje de un $8,6 \%$, apareciendo entre 4 y $14 \%$ en dos estudios donde se especifica ${ }^{6,11}$.

En este estudio, a diferencia de la mayoría de artículos revisados, se ha distinguido entre persistencia y reaparición del dolor tras la cirugía, ya que las causas que pueden originar cada uno de ellos son diferentes tal y como se refleja en el artículo de Fritsch et al. ${ }^{11}$ en el que se señalan, como causas más frecuentes de persistencia del dolor, la cirugía de un segmento erróneo, la extirpación insuficiente de la hernia discal, la existencia de una segunda hernia no reconocida, la lesión de la raíz nerviosa y la insuficiente descompresión de la estenosis del canal raquídeo. Respecto a la reaparición del dolor, las causas reflejadas en dicho artículo son la recurrencia de la misma hernia, aparición de otra hernia a distinto nivel, fibrosis peridural, síndrome facetario, inestabilidad y espondilodiscitis.

El porcentaje de persistencia del dolor en la muestra fue del $10,15 \%$, que no se ha podido comparar con los trabajos revisados, ya que no precisan este dato; un $31,9 \%$ de los pacientes presentaron reaparición del dolor, de los cuales en un $40,5 \%$ se produjo entre 1 y 3 meses tras la cirugía, período señalado en la literatura como clave cuando la etiología del mismo es la fibrosis peridural, responsable hasta en un $24 \%$ de los casos de la cirugía fallida de hernia discal lumbar ${ }^{14,19,22}$. En el estudio prospectivo de Ross et al. ${ }^{19}$ con 197 pacientes encontraron porcentajes de un $22 \%$ de dolor postquirúrgico cuando no utilizaron gel barrera antiadherente Adcon-L y un 16,7\% cuando lo utilizaron; en el resto de los artículos revisados no se menciona la reaparición del dolor como tal, sino que hacen referencia a buen o mal resultado, y al alivio parcial o completo del dolor.

En 27 casos de los pacientes se ha solicitado RM en el seguimiento postquirúrgico ante la aparición del SCRF, encontrando en 11 de ellos fibrosis peridural, en 8 recidiva de la misma hernia y en 5 casos ambos hallazgos radiológicos. Por tanto, de los que se realizaron RM, casi un $60 \%$ presentaban fibrosis peridural, y sólo en 3 casos no se encontraron hallazgos que justificasen el dolor.

Desde el punto de vista sociolaboral, se ha encontrado que los pacientes de la muestra están en su mayoría casa- 
dos $(72,5 \%)$ y con un bajo nivel de estudios (sin estudios o primarios, en un $67,1 \%$ ), no hallando estudios que especifiquen estos parámetros para comparar resultados. Respecto a la situación laboral previa a la cirugía un $68,1 \%$ de los pacientes de la muestra se encontraban activos laboralmente, un $15,4 \%$ eran pensionistas y un $12,1 \%$ eran amas de casa. De los que se encontraban en el mercado laboral, destaca un $24,2 \%$ de pacientes dedicados al sector agrícola, y similares cifras en la hostelería y conducción $(9,7 \%$ en cada uno). Un 58\% referirían coger peso habitualmente en su trabajo. No se dispone de datos en los artículos encontrados para comparar la situación laboral previa. La reincorporación laboral a medio plazo (entre 6 y 12 meses), fue de un $64,5 \%$, y en más de la mitad estos casos se llevó a cabo con más de 3 meses tras la cirugía; se han seleccionado de forma arbitraria los períodos, menos de 3 semanas, entre 3 semanas y 3 meses, y más de 3 meses, ya que en los artículos revisados se encontró importante heterogeneidad en este punto. Estos resultados son similares a los obtenidos por Harold et al. ${ }^{2}$ en su estudio prospectivo con 212 seguimiento de dos años, donde un $61 \%$ de los pacientes se reincorporó al trabajo. Sin embargo, los resultados obtenidos en este punto son notablemente inferiores; comparándolos con otros estudios a medio plazo, como el retrospectivo realizado por Donceel et al. ${ }^{10}$ con 177 pacientes, donde la reincorporación laboral al año ascendía al 85\%; así como los estudios prospectivos de Atlas et al. ${ }^{3}$ y Nygaard et al. ${ }^{16}$ donde el $95 \%$ de 275 pacientes y el $80 \%$ de 132 pacientes respectivamente, se reincorporaron laboralmente tras someterse a cirugía de hernia discal.

En nuestra serie, sólo un 33\% de los pacientes del estudio había realizado algún tipo de tratamiento rehabilitador tras la cirugía, y en estos, el porcentaje de cirugía fallida fue casi el doble (66\%) respecto a los que no la hicieron (34\%); este resultado no es valorable, si se tiene en consideración que los pacientes enviados al servicio de Rehabilitación son aquellos con peores resultados quirúrgicos. El porcentaje de pacientes enviados a realizar tratamiento rehabilitador es muy escaso, si se considera la revisión Cochrane recientemente publicada con 13 estudios (6 de alta calidad) llevada a cabo por Ostelo et al. ${ }^{18}$, que concluye que existe una fuerte evidencia de que un programa intensivo de ejercicios, al menos si empiezan entre la $4^{\mathrm{a}}$ y la $6^{\mathrm{a}}$ semana postcirugía, mejora a corto plazo el estado funcional del paciente y favorece una reincorporación laboral más temprana. El contenido exacto del programa rehabilitador no está muy claro según esta revisión, al igual que tampoco queda claro si debe empezarse inmediatamente tras la cirugía o entre 4 y 6 semanas después. No existe evidencia de que los pacientes necesiten restringir sus actividades tras someterse por primera vez a cirugía de hernia discal. En esta línea, Carragee et al. ${ }^{5}$, en su estudio prospectivo con 152 pacientes sometidos a discectomia lumbar, animaban a los pacientes a retornar a sus actividades habituales tan pronto como pudieran, eliminando cualquier tipo de restricción postquirúrgica, consiguiendo acortar el tiempo de reincorporación laboral que, además, se alcanzó en un $98 \%$. Las complicaciones que aparecieron fueron similares a las reportadas en la literatura para pacientes donde se aplicaron restricciones tras la cirugía. Por todo ello, parece claro que las limitaciones y restricciones postcirugía discal lumbar no están basadas en hallazgos clínicos, y refleja el miedo del terapeuta a provocar recidiva herniaria, daño o inestabilidad con la no restricción de actividades?.

La satisfacción de los pacientes de la muestra en nuestra serie fue de 79,1\% con la cirugía, algo inferior al estudió de Atlas et al. ${ }^{3}$ donde encontraron que un $87,5 \%$ de los 275 pacientes volverían a elegir la cirugía como opción terapéutica. En el resto de los trabajos revisados, los porcentajes que se obtienen de satisfacción son similares, oscilando entre el 76 y $89 \%^{2,13,15}$. Es de destacar que 3 de cada 4 pacientes que presentaron cirugía fallida en nuestro estudio no se muestran insatisfechos con la opción elegida, lo que hace suponer que los pacientes estaban bien informados antes de la intervención de los inconvenientes y dificultades de este tipo de cirugía.

Somos conscientes que en lo relativo a la calidad de vida, lo ideal hubiera sido comparar las distintas dimensiones del cuestionario de salud SF-36 en el momento previo y posterior a la cirugía, para tratar de establecer hasta qué punto la calidad de vida de los pacientes mejora tras someterse a cirugía de hernia discal lumbar. Sin embargo, dado el carácter descriptivo transversal del estudio no se ha podido comparar este aspecto, aunque se ha encontrado que la calidad de vida está claramente afectada en todos los aspectos del SF-36 en aquellos que presentaron cirugía fallida tras intervención de hernia discal lumbar, con significación estadística en 4 de ellas. En este sentido, se han encontrado dos estudios prospectivos que concluyen que la calidad es mejor con la cirugía. Atlas et al. ${ }^{3}$ utilizan el SF36 mientras que González-Darder et al. ${ }^{12}$ emplean el Perfil de Salud Nottingham.

Para concluir la discusión, se analizan los resultados de la relación de cirugía fallida con el resto de parámetros en los pacientes sometidos a primera cirugía de hernia discal lumbar:

a) No se ha encontrado relación de la cirugía fallida con el sexo, con la edad, con las complicaciones postquirúrgicas y con cargar peso habitualmente en el trabajo.

b) Se ha hallado una cierta relación de la cirugía fallida con la ciática derecha, con un tiempo de evolución del dolor prequirúrgico superior a 6 meses, con los niveles de hernia discal L4-L5, con la fibrosis peridural en RM, con el nivel de estudios primario y universitario, con el estado civil casado, con los activos laboralmente antes de la cirugía, con el trabajo en el sector agrícola y con los que pro- 
longaron la baja laboral por más de 3 meses tras la cirugía.

c) Se ha encontrado una fuerte relación de la cirugía fallida con la clínica precirugía de ciática bilateral, con la asociación de estenosis de canal a la hernia discal, con la comorbilidad (hipertensión, diabetes, cardiopatía y dislipemia), con pacientes sin estudios, con el trabajo del sector de la conducción y algo menos en el sector de la construcción y hostelería, y finalmente con la situación de baja o invalidez tras la cirugía.

d) Se encuentra significación estadística de la cirugía fallida con la satisfacción del paciente y con la calidad de vida en las dimensiones del SF-36 de función física, dolor corporal, vitalidad y rol emocional. Por tanto, el perfil del paciente con cirugía fallida teniendo en cuenta la significación estadística, sería el de una persona limitada para actividades físicas, afectado por dolor hasta el punto de repercutir y limitar las actividades fuera de casa y en el hogar, con sensación frecuente de cansancio o agotamiento, y cuyos problemas emocionales interfieren en su trabajo y actividades de la vida diaria.

Un punto que se ha considerado importante es el tiempo de evolución del dolor precirugía. Los resultados de este estudio están en la línea, aunque no de forma significativa, de aquellos autores que, de forma significativa, concluyen en sus trabajos que cuanto mayor sea la historia de evolución del dolor antes de la intervención mayores son las probabilidades de resultados desfavorables o cirugía fallida ${ }^{1,21,23}$; a diferencia del estudio de Fritsch et al. ${ }^{11}$ donde no encontraron relación entre cirugía fallida y larga evolución del dolor. Al igual que Vogelsang et al. ${ }^{22}$ encontramos que la fibrosis peridural es un valor menor en el diagnóstico diferencial del dolor recurrente tras discectomía lumbar. Para finalizar, añadir que se ha coincidido con el estudio de Woertgen $\mathrm{C}$ et al. ${ }^{15}$ cuando señalan que factores predictivos negativos de la cirugía de hernia discal lumbar son nivel educacional bajo y trabajos como la agricultura, encontrando además en nuestro estudio peores resultados en los trabajadores de la hostelería, construcción, y especialmente en los de la conducción. En este sentido, aunque uno puede suponer que el nivel educacional bajo se correlaciona con cirugía fallida por el tipo de trabajo desempeñado, más físico, en relación a aquellos con estudios superiores, en nuestra serie no encontramos relación con cargar o no cargar peso en su actividad laboral diaria, y, además, hemos obtenido el mismo porcentaje del SCRF en pacientes con estudios primarios y aquellos con estudios universitarios.

\section{Agradecimientos}

Queremos agradecer al Servicio de Rehabilitación de los HHUU Virgen del Rocío la colaboración y facilidades prestadas para poder llevar a cabo este trabajo, y como no, a todos los pacientes que hemos entrevistado telefónicamente, sin cuya colaboración desinteresada no hubiera sido posible realizar este estudio.

\section{Bibliografía}

1. Alonso, J., Prieto, L., Anto, J.M.: La versión española del SF-36 Health Survey (Cuestionario de Salud SF-36): un instrumento para la medida de los resultados clínicos. Medicina Clínica 1995; 104: 771-776.

2. Asch, H.L., Lewis, P.J., Moreland, D.B., et al.: Prospective multiple outcomes study of outpatient lumbar microdiscectomy: should 75 to $80 \%$ succes rates be the norm? J Neurosurg. (Spine 1) 2002; 96: 34-44.

3. Atlas, S.J., Deyo, R.A., Keller, R.B., et al.: The maine lumbar spine study, part II: 1-year outcomes of surgical and nonsurgical management of sciatica. Spine 1996; 21: 1777-1786.

4. Badia, X.., Salaveo; M., Alonso, J.: La medida de la Salud. Guías de Escalas de medición en español. $3^{a}$ Edición. Barcelona; Fundación Lilly, 2002; pp. 196-204.

5. Carrage, E.J., Han, M.Y., Yang, B., Kim. D.H., Kraemer. H., Billys, J.: Activity restrictions after posterior lumbar discectomy: A prospective study of outcome in 152 cases with no postoperative restrictions. Spine 1999; 24: 2346-2355.

6. Davis, R.A.: A long-term outcome analysis of 984 surgically treated herniated lumbar discs. J Neurosurg. 1994; 80: 415-421.

7. De Groot, K., Boeke, S., Passchier, J.: Preoperative expectations of pain and recovery in relation to postoperative disappointment in patients undergoing lumbar surgery. Medical Care 1999 ; 37: 149-156.

8. Donceel, P., Du Bois, M.: Fitness for work after surgery for lumbar disc herniation: a retrospective study. Eur Spine J 1998; 7: 29-35.

9. Donceel, P., Du Bois, M,. Lahaye, D.: Return to work after surgery for lumbar disc herniation: A rehabilitationoriented approach in insurance medicine. Spine 1999; 24: 872-879.

10. Donceel, P., Du Bois, M.: Predictors for work incapacity continuing after disc surgery. Sean J Work Environ Health 1999; 25:264-271.

11. Fritsch, E.W., Heisel, J., Rupp, S.: The failed back surgery symdrome: reasons, intraoperative findings, and longterm results: a report of 182 operative treatment. Spine 1996; 21: 626-633.

12. González-Darder., J.M., Torres-Recio, J.: Calidad de vida tras el tratamiento quirúrgico de la hernia discal lumbar. Estudio prospectivo con un año de seguimiento. Mapfre Medicina 1995 ; 6 : 85-89.

13. Haglund, M.M.. Moore, A.J., Marsh; H., Uttley, D.: Outcome after repeat lumbar microdiscectomy. Br J Neurosurgery 1995; 9: 487-495.

14. Isla, A., Alvarez, F.: Spinal epidural fibrosis following lumbar discectomy and antiadhesion barrier. Neurocirugía 
$2001 ; 12: 439-446$.

15. Loupasis, G.A., Stamos, K., Katonis, P.G., Sapkas, G., Korres, D.S., Hartofilakidis, G.: Seven - to 20 - year outcome of lumbar discectomy. Spine 1999; 24: 2313-2317.

16. Nygaard, O.P., Kloster. R., Solbera. T.: Duration of leg pain as a predictor of outcome after surgery for lumbar disc herniation: A prospective cohort study with 1-year follow up. J Neurosurgery (Spine 2) 2000; 92: 131-134.

17. Ostelo. R.W.J.G., de Vet, H.C.W., Waddell., G., Kerckhoffs., M.R., Leffers., P., van Tulder, M.W.: Rehabilitation following first-time lumbar disc surgery: a systematic review within the framework of The Cochrane Colaboration. Spine 2003; 28: 209-218.

18. Ostelo, R.W.J.G., de Vet, H.C.W., Waddell, G., Kerckhoffs, M.R., Leffers, P., van Tulder, M.W.: Rehabilitation after lumbar disc surgery (Cochrane Review). In: The Cochrane Library, Issue 1, 2004. Chichester, UK: John Wiley \& Sons, Ltd.

19. Ross. J.S., Robertson, J.T., Frederickson, R.C.A., et al.: Association between peridural star and recurrent radicular pain after lumbar discectomy: magnetic resonante evaluation. Neurosurgery 1996; 38: 855-863.

20. Schade, V.. Semmer.. N., Main; C.J., Hora., J., Boss., N.: The impact of clinical, morphological, psychosocial and work-related factors on the outcome of lumbar discectomy. Pain 1999; 80: 239-249.

\section{Comentario al trabajo: Factores relacionados con la cirugía fallida de hernia discal lumbar de Rodríguez García y cols.}

El articulo tiene interés por ser valorado por una especialidad como es Rehabilitación que tan asociada está a la especialidad de Neurocirugía. Uno de los factores más importantes de este trabajo es la reincorporación laboral de los pacientes en edad activa después de la cirugía de hernia discal lumbar, y que lo observaron en un $64.5 \%$ de los casos que fueron intervenidos quirúrgicamente. Hay que reseñar que un porcentaje de los pacientes incluidos en el estudio habían sido previamente intervenidos de hernia discal y lógicamente incrementa el porcentaje de cirugía fallida como los autores refieren. Por otra parte, llama la atención que, con respecto al grado de satisfacción de los pacientes después de la cirugía, el 79,1 \% afirmó que elegirían de nuevo la opción quirúrgica frente a un $11 \%$ que no la elegirían de nuevo, o a un 9.9\% dudoso. Esta diferencia en relación al porcentaje general de cirugía fallida no sólo está en relación con que estén bien informados los pacientes de la cirugía que se les iba a realizar como comentan los autores, sino también con ese porcentaje de pacientes que pueden entrar en el tema conflictivo de "neurosis de renta" que en principio han aceptado los resultados de la cirugía
2005; 16: 507-517

21. Schoeggl, A., Maier, H., Saringer, W., Reddy,M., Matula, C.: Outcome after chronic sciatica as the only reason for lumbar microdiscectomy. J Spinal Disord Tech. 2002; 15: 415-419.

22. Vogelsang, J.P., Finkenstaedt, M., Vogelsang, M., Markakis, E.: Recurrent pain after lumbar discectomy: the diagnostic value of peridural star on MRI. Eur Spine J 1999; 8: 475-479.

23. Woertgen,C.. Holzschuh, M.. Rothoerl, R.D., Brawanski, A.: Does the choice of outcome scale influence prognostic factors for lumbar disc surgery? A prospective, consecutive study of 121 patients. Eur Spine J 1997; 6:173-180.

Este trabajo ha sido presentado en el 42 Congreso de la Sociedad Española de Rehabilitación y Medicina Física (SERMEF) celebrado en La Coruña los días 8-11 de Junio de 2004, obteniendo una beca-premio al segundo mejor trabajo nacional realizado por residentes.

Rodríguez-García, J.; Sánchez-Gastaldo, A.; Ibáñez-Campos, T.; Vázquez-Sousa, C.; Cantador-Hornero, M.; ExpósitoTirado, J.A.; Cayuela-Domínguez, A.; Echevarría-Ruiz de Vargas, C.: Factores relacionados con la cirugía fallida de hernia discal lumbar. Neurocirugía 2005; 16: 507-517.

Correspondencia postal: Jorge Rodríguez García. Avd/ De las Ciencias $n^{\circ} 10$, Blq. $7,6^{\circ}$ D. 41020 . Sevilla.

pero que cabe la duda que no señalen la realidad de su estado clínico. Hubiera sido interesante haber ahondado un poco más en los casos en los que la cirugía no fue bien, pero quiero destacar que la fibrosis que se observó en los casos de reintervención tiene una relativa importancia ${ }^{1}$ o quizás ninguna como afirman los autores. También quiero comentar, y de una forma algo mas crítica, que la escala SF- 36 de valoración clínica es eficaz, pero creo que para una entrevista telefónica es complicada y tediosa, con riesgo de que un paciente no pueda contestarla de forma adecuada. Se pueden utilizar otras escalas de valoración como es la de Prolo $^{2}$ si se utiliza este medio. En definitiva, creo que es un artículo de interés para la revista Neurocirugía.

\section{Bibliografía}

1. Isla, A., Alvarez, F.: Fibrosís epidural postdiscectomía lumbar y barrera antihadesiva. Neurocirugía 2001;12: 439-446.

2. Prolo. D.J., Oklund, S.A., Butcher, M.: Toward uniformity in evaluating results of lumbar of lumbar spine operations: a paradigm applied to posterior lumbar interbody fusion. Spine 1986;11:601-606. 